\title{
Direct and quasi-direct band gap silicon allotropes with remarkable stability
}

\author{
Chaoyu He, ${ }^{1,2}$ Jin Li, ${ }^{1,2}$ xiangyang Peng, ${ }^{1,2}$ Lijun Meng,,${ }^{1,2}$ Chao Tang,,${ }^{1,2}$ and Jianxin Zhong ${ }^{1,2, \text { 用 }}$ \\ ${ }^{1}$ Hunan Key Laboratory for Micro-Nano Energy Materials and Devices, Xiangtan University, Hunan 411105, P. R. China; \\ ${ }^{2}$ School of Physics and Optoelectronics, Xiangtan University, Xiangtan 411105, China.
}

(Dated: October 1, 2018)

\begin{abstract}
In our present work, five previously proposed $\mathrm{sp}^{3}$ carbon crystals were suggested as silicon allotropes and their stabilities, electronic and optical properties were investigated by first-principles method. We find that these allotropes with direct or quasi-direct band gaps in range of 1.2-1.6 eV are very suitable for applications in thin-film solar cells. They display strong adsorption coefficients in the visible range of the sunlight in comparison with diamond silicon. These five silicon allotropes are confirmed possessing positive dynamical stability and remarkable themodynamical stability close to that of diamond silicon. Especially, the direct band gap M585-silicon possessing energy higher than diamond silicon only $25 \mathrm{meV}$ per atom is expected to be experimentally produced for thin-film solar cells.
\end{abstract}

PACS numbers: 78.20.Ci, 42.79.Ek, 71.10.-w, 88.40.jj

Silicon is becoming the fundamental element in our daily life. Almost all the semiconductor components in high technology of today are silicon-based. As the second most aboundant element in earth crust, silicon possesses many advantages not only for applications in the semiconductor industry. It is also the leading material foundation in the important field of photovoltaic energy production. Due to its abundance and ability to translate the solar energy, as well as the reducing non-renewable traditional energy, silicon is believed as the future of human. Theoretically, silicon can forms many allotropes due to its ability of $\mathrm{sp}^{3}$ hybridization like carbon. But, unfortunately, it is always locked in oxide or lie in its diamond like ground state.

Although the band gap of diamond silicon of about 1.12 $\mathrm{eV}^{\underline{1}}$ lies in the optimal adsorption range of the sunlight, its indirect characteristic seriously affects its adsorption efficiency ${ }^{2}$. Thus, many efforts have been paid on searching for viable silicon allotropes with direct or quasi-direct band gaps overlaping the sunlight in visible range, for the purpose of improving the translating efficiency of the solar energy. For example, Botti et al. have proposed some low-energy silicon allotropes with quasi-direct band gaps in the range of 0.8-1.5 $\mathrm{eV}$ and possessing strong adsorption coefficients for thin-film solar cell applications ${ }^{3}$. Also, an approach named as Inverse Band Structure Design Approach (IBSDA) based on the particle swarming optimization algorithm ( $\mathrm{PSO}^{4}$ ) was developed by Xiang et al. for the purpose of searching for materials with expected electronic properties and a quasi-direct band gap silicon allotrope $\mathrm{Si}_{20}$-T was predicted $\stackrel{5}{5}$. Based on the IBSDA as implemented in PSO, Wang et al. have also found six low energy direct and quasi-direct silicon allotropes ${ }^{6}$. Very recent, Lee et al. suggested another approach to search for direct band gap silicon allotropes and they successfully discovered many direct and quasi-direct band gap allotropes ${ }^{T}$. Especially, a recent experimental progress shows that a cage-like silicon allotrope $\mathrm{Si}_{24}$ with a quasi-direct band gap of about $1.3 \mathrm{eV}$ can be synthesized by Na-assistanted two step approach ${ }^{8}$. The crystal structure is confirmed as the same as that of the previously suggested $\mathrm{CAS}-\mathrm{Si}^{9}$. The authors also show that $\mathrm{Si}_{24}$ possesses strong adsorption coefficient higher than
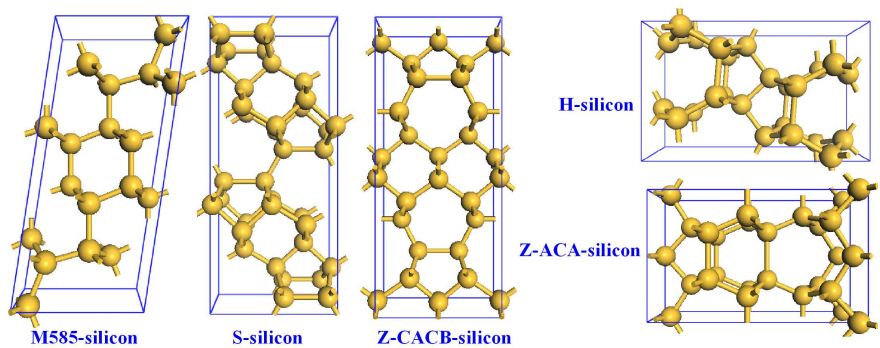

FIG. 1: Perspective view of the optimized crystal structues of M585silicon, S-silicon, Z-CACB-silicon, H-silicon and Z-ACA-silicon.

diamond silicon by first-principles calculation ${ }^{8}$. All these theoretical successful examples make us in confident in using computational-alchemy in the engineering of materials 10 .

In this paper, we predict five low energy silicon allotropes (M585-silicon, S-silicon, Z-CACB-silicon, H-silicon and Z-ACA-silicon as shown in Fig. 1) based on the crystal structures of our previously proposed $\mathrm{sp}^{3}$ carbon phases ${ }^{11,12}$ and find that they are direct or quasi-direct band gap semiconductors with strong adsorption coefficients for thin-film solar cell application. Such a transplanting idea of crystal structures have made a lot of successes in crystal prediction, such as zeolite nets to carbon allotropes $\frac{13}{13}$ and carbon crystals to silicon allotropes like Bct-C4 silicon-14,15, M4 silicon노,16, $\mathrm{M} 10$ silicon ${ }^{\frac{3}{}}$ and $\mathrm{Cco}-\mathrm{Si} 8^{3,17-19}$. Our calculating results show that these five silicon allotropes possess positive dynamical stability and remarkable themodynamical stability comparable to that of diamond silicon. Especially, the direct band gap M585-silicon possessing energy higher than diamond silicon only $25 \mathrm{meV}$ per atom is expected to be experimentally produced for thin-film solar cells.

In our present work, we perform the structure optimizations and total energy calculations using the density functional theory within local density approximation (LDA) ${ }^{20,21}$ as implemented in Vienna ab initio simulation package (VASP) 22,23. The interactions between nucleus and the $3 \mathrm{~s}^{2} 3 \mathrm{p}^{2}$ valence electrons of silicon are described by the 
TABLE I: Total energies, energy band gaps and fundamental structural information including space group, lattice constants and mass densities of diamond silicon, M585-silicon, S-silicon, Z-CACB-silicon, H-silicon and Z-ACA-silicon.

\begin{tabular}{ccccccc}
\hline \hline Items & Diamond silicon & M585-silicon & S-silicon & Z-CACB-silicon & H-silicon & Z-ACA-silicon \\
\hline Space group & FD-3M (No.227) & P21/M (No.11) & CMCM (No.63) & IMMA (No.74) & PBAM (No.55) PMMN (No.59) \\
Lattice a & $5.407 \AA$ & $14.64 \AA$ & $3.825 \AA$ & $3.833 \AA$ & $11.832 \AA$ & $3.807 \AA$ \\
Lattice b & $5.407 \AA$ & $3.814 \AA$ & $17.141 \AA$ & $7.289 \AA$ & $7.234 \AA$ & $7.055 \AA$ \\
Lattice c & $5.407 \AA$ & $6.772 \AA$ & $7.399 \AA$ & $17.41 \AA$ & $3.812 \AA$ & $12.002 \AA$ \\
Mass density & $2.361 \mathrm{Mg} / \mathrm{cm}^{3}$ & $2.311 \mathrm{Mg} / \mathrm{cm}^{3}$ & $2.307 \mathrm{Mg} / \mathrm{cm}^{3}$ & $2.302 \mathrm{Mg} / \mathrm{cm}^{3}$ & $2.284 \mathrm{Mg} / \mathrm{cm}^{3}$ & $2.315 \mathrm{Mg} / \mathrm{cm}^{3}$ \\
Relative energy & 0 & $25 \mathrm{meV} / \mathrm{atom}$ & $42 \mathrm{meV} / \mathrm{atom}$ & $70 \mathrm{meV} / \mathrm{atom}$ & $61 \mathrm{meV} / \mathrm{atom}$ & $78 \mathrm{meV} / \mathrm{atom}$ \\
Band gap & $1.11 \mathrm{eV}$ & $1.51 \mathrm{eV}$ & $1.41 \mathrm{eV}$ & $1.33 \mathrm{eV}$ & $1.52 \mathrm{eV}$ & $1.29 \mathrm{eV}$ \\
Direct band gap & $3.32 \mathrm{eV}$ & $1.51 \mathrm{eV}$ & $1.53 \mathrm{eV}$ & $1.38 \mathrm{eV}$ & $1.63 \mathrm{eV}$ & $1.43 \mathrm{eV}$ \\
\hline \hline
\end{tabular}

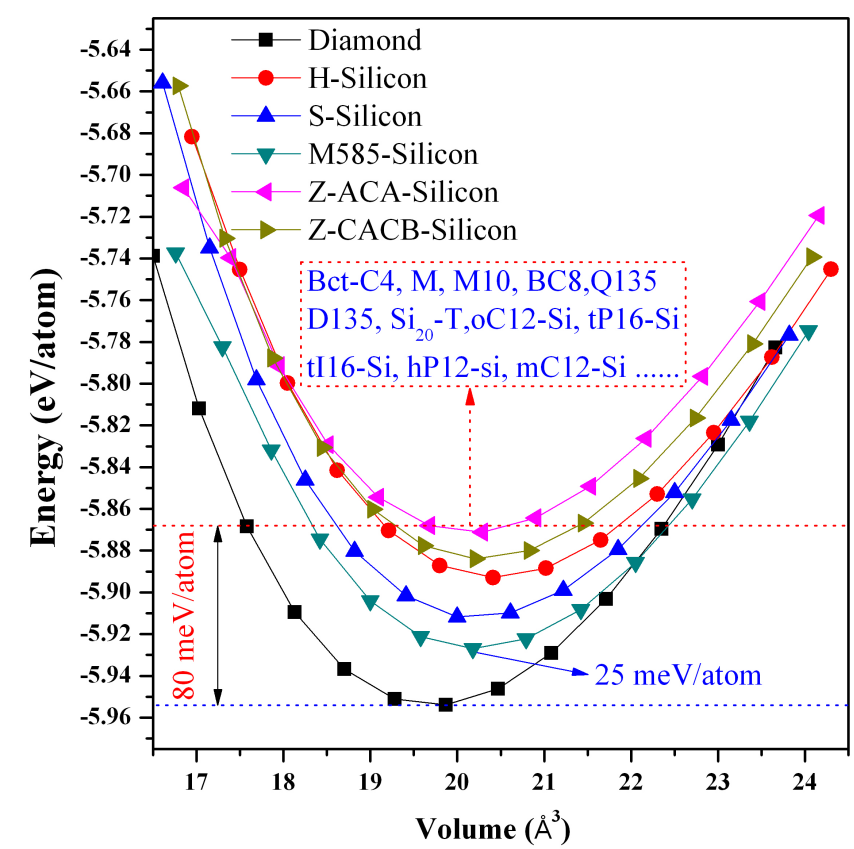

FIG. 2: Energy per atom as a function of volume per atom for diamond silicon, M585-silicon, S-silicon, Z-CACB-silicon, H-silicon and Z-ACA-silicon.

projector augmented wave (PAW) method ${ }^{24,25}$. A plane-wave basis with a cutoff energy of $400 \mathrm{eV}$ is used to expand the wave functions and the Brillouin Zone (BZ) sample meshes are set to be dense enough (less than $0.21 \AA^{-1}$ ) to ensure the accuracy of our calculations. All the crystal structures of silicon allotropes considered in our present work are fully optimized up to the residual force on every atom less than $0.005 \mathrm{eV} / \AA$. The dynamical stabilities of the five new silicon allotropes were evaluated through simulating their vibrational properties by using the PHONON package 26 with the forces calculated from VASP. Especially, the hybrid functional method (HSE06) ${ }^{27}$ is also considered to accurately calculate the electronic and optical properties of the five new silicon allotropes.

As shown in Fig. 1 is the optimized crystal structures of M585-silicon, S-silicon, Z-CACB-silicon, H-silicon and Z-ACA-silicon. Their relative energy, energy band gaps, as well as fundamental structural information including space groups, lattice constants, mass densities are summarized in Table I , those of diamond silicon are also listed for comparison. We can see that the mass density of M585-silicon, S-silicon, Z-CACB-silicon, H-silicon and Z-ACA-silicon are $2.311 \mathrm{Mg} / \mathrm{cm}^{3}, 2.307 \mathrm{Mg} / \mathrm{cm}^{3}, 2.302 \mathrm{Mg} / \mathrm{cm}^{3}, 2.284 \mathrm{Mg} / \mathrm{cm}^{3}$ and $2.315 \mathrm{Mg} / \mathrm{cm}^{3}$, respectively. These results indicate that M585-silicon, S-silicon, Z-CACB-silicon, H-silicon and $\mathrm{Z}$-ACA-silicon are dense close to diamond silicon, which is good consistent with their $\mathrm{sp}^{3}$ configurations.

From the total energies as listed in Table I and shown in Fig. 2, we find that all these five silicon allotropes are metastable with energies higher than that of diamond silicon in $80 \mathrm{meV}$ per atom. That is to say, they are themodynamically favorable than most of the previously proposed silicon allotropes, such as the quasi-direct band gap $\mathrm{Si}_{20}-\mathrm{T}^{5}$ and Q135 ${ }^{-}$as well as the direct band gap D1357 and mC12-Si ${ }^{-6}$. In Fig. 2, we also show the total energy per atom as a function of volume per atom for these new silicon allotropes (Results about other phase with energies higher than diamond silicon up to $80 \mathrm{meV}$ per are not shown in it for the considering of simplification). From the quadratic $\mathrm{E}-\mathrm{V}$ relation near the equilibrium $\mathrm{V}_{0}$, we can see that all of them are themodynamically stable protected by obvious energy barriers in their individual local state. From Table I and Fig. 2, we can easily know that the most stable one among these five silicon allotropes is M585-silicon, followed by S-silicon and H-silicon. Its energy is just $25 \mathrm{meV}$ per atom higher than that of diamond silicon, which indicates that it possesses the very high probability to be synthesized in experiment.

To further confirm the dynamical stabilities of these five new silicon allotropes, we then investigated their vibrational properties through simulation their phonon band structures and density of state. Their phonon band structures are shown in Fig. 3. According to our results, there is no any imaginary frequency appearing in these phonon vibrational spectra and no any imaginary models appearing it their phonon density of state. Such vibrational properties indicate that these five new silicon allotropes are dynamically stable.

As mentioned before, direct or quasi-direct band gap characteristics are expected for sunlight adsorption, which can avoiding the requirement of thick absorber layer (for indirect band gap semiconductors such as diamond silicon) to provide necessary phonon moment. Thus, we firstly care about the electronic band structures of these five promising silicon 

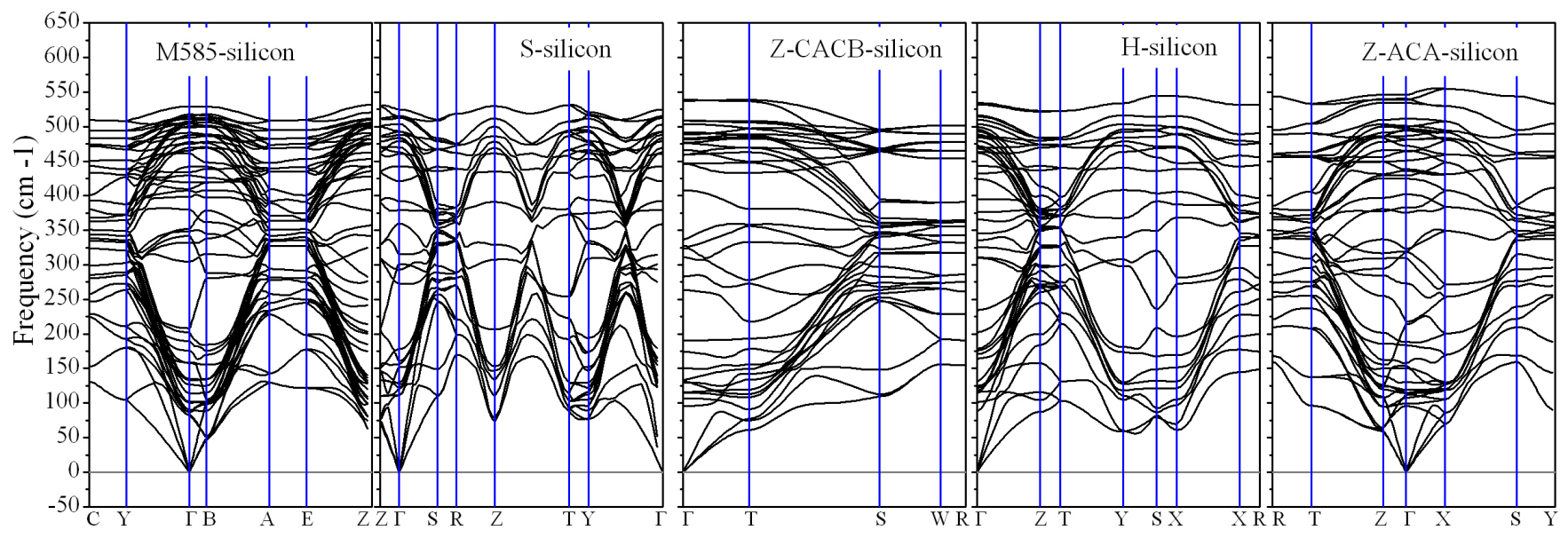

FIG. 3: Phonon band structures of M585-silicon, S-silicon, Z-CACB-silicon, H-silicon and Z-ACA-silicon.

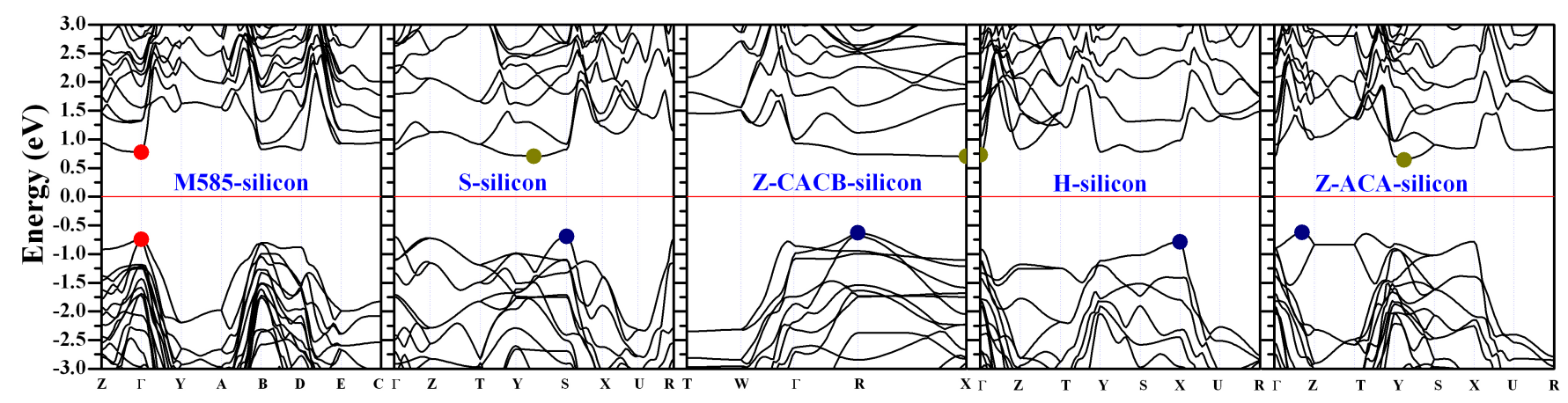

FIG. 4: Electronic band structures of M585-silicon, S-silicon, Z-CACB-silicon, H-silicon and Z-ACA-silicon.

allotropes. Considering that traditional DFT-method always underestimate the band gap of semiconductor in comparison of the experiment results, we employ the HSE06 method in our present work to investigate the electronic properties of M585-silicon, S-silicon, Z-CACB-silicon, H-silicon and Z-ACA-silicon. The calculated band structures are shown in Fig. 4 and corresponding band gaps are summarized in Table I. From the band structures we can see that the most stable M585-silicon is a typical direct band gap semiconductor with a gap of $1.51 \mathrm{eV}$. S-silicon, Z-CACB-silicon, H-silicon and Z-ACA-silicon behave as quasi-direct band gap semiconductors with band gaps of $1.41 \mathrm{eV}, 1.29 \mathrm{eV}, 1.33 \mathrm{eV}$ and $1.52 \mathrm{eV}$, respectively. Their corresponding direct band gaps are 1.53 $\mathrm{eV}, 1.38 \mathrm{eV}, 1.63 \mathrm{eV}$ and $1.43 \mathrm{eV}$, respectively. These results indicate that they are suitable for applications in thin-film solar cells as absorbers.

We then turn our interesting on the absorption abilities of these five promising silicon allotropes. Absorption spectra of these allotropes are also calculated based on HSE06 method. The results are shown in Fig. 5 in comparison with that of the diamond silicon and the air mass 1.5 solar spectral irradiance ${ }^{28}$. We can see that all of these five silicon allotropes possess strong absorption coefficients better than that of diamond silicon in the energy range between 1.5

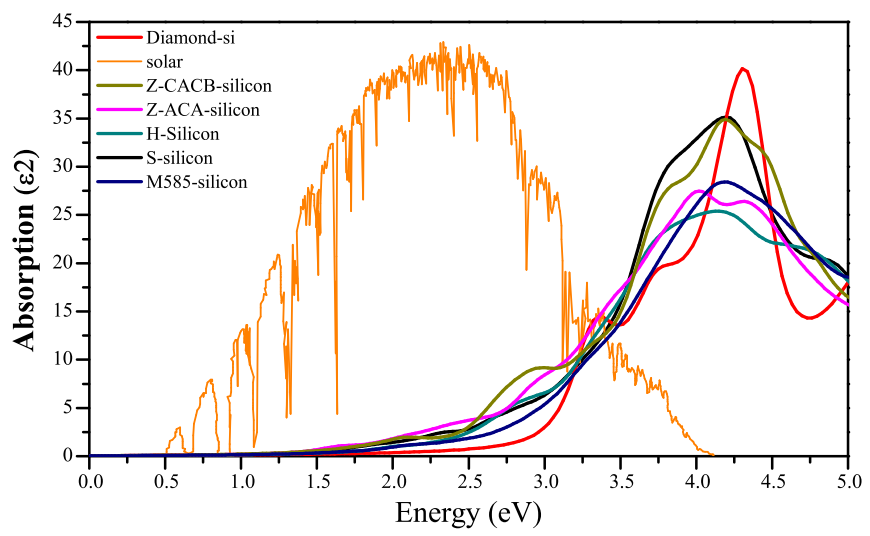

FIG. 5: Adsorption spectra of M585-silicon, S-silicon, Z-CACBsilicon, H-silicon and Z-ACA-silicon compared to that of diamond silicon and the reference air mass 1.5 solar spectral irradiance ${ }^{28}$.

and $3.2 \mathrm{eV}$. It is well known that indirect band gap diamond silicon needs necessary phonon moments to assist its indirect band adsorption 2 . Thus it is always prepared with thickness about $100 \mu \mathrm{m}$ to ensure the adsorption of solar energy below 
its direct optical gaps 29 . Our discoveries suggest that these five new silicon allotropes are suitable for thin-film solar cell applications in views of their direct or quasi-direct band gap characteristics and strong adsorption abilities, if they can be successfully synthesized in future experiment.

Usually, silicon is always locked in oxide or lies in its ground state of diamond silicon. There is no any graphite-like silicon allotrope that can provides abundant pathways to many other metastable phases under high pressure condition like carbon ${ }^{30}$. Although diamond silicon can also undergos a series of phase transitions 31,32 with the increase of external pressure, from diamond to $\beta$-Sn at about $12 \mathrm{Gpa}$, from $\beta$-Sn to orthorhombic Imma phase and then to simple hexagonal at about 13-16 Gpa, from simple hexagonal to an orthorhombic Cmca phase at about $38 \mathrm{Gpa}$, from Cmca to a new hexagonal close pack at 42 Gpa and finally to face-centered cubic at 78 Gpa , all these high pressure phases are metallic and non of them can keep existing at ambient condition. Fortunately, the process of pressure-release of $\beta$-Sn phase provides us many opportunities to meet some metastable silicon allotropes 33 , such as R8, BC8 and other unknown phases ${ }^{32}$ with distorted $\mathrm{sp}^{3}$ bondings, depending on the release velocity and temperature condition. We expect further experiments in future can find out proper release velocity and temperature conditions to synthesize these low energy silicon allotropes based on the high pressure $\beta$-Sn phase. On the other hand, other experimental methods have also been developed to synthesize new metastable silicon allotropes 8,34,35. M585-silicon, S-silicon, Z-CACB-silicon, H-silicon and Z-ACA-silicon are topological similar to the recently synthesized allo-Ge phases, which indicate that they may be synthesized by similar method 35 . The recent success of synthesizing of a cage-like silicon allotrope $\mathrm{Si}_{24}$ with a quasi-direct band gap of about $1.3 \mathrm{eV}$ by $\mathrm{Na}$-assistanted two step approach ${ }^{8}$ also provides a suitable method for synthesizing the five new silicon phases suggested in our present work. Especially, the M585-silicon with the lowest energy (about $80 \mathrm{meV}$ lower than that of $\mathrm{Si}_{24}$ ) and cage-like configuration similar to that of $\mathrm{Si}_{24}$ is expected to be synthesized as the first experimental direct band gap silicon for thin-film solar cell application.

In summary, five previously proposed $\mathrm{sp}^{3}$ carbon crystals were theoretically suggested as silicon allotropes for the first time and their stabilities, electronic and optical properties were investigated by first-principles method. We find that these allotropes with direct or quasi-direct band gaps in range of 1.2-1.6 eV are very suitable for applications in thin-film solar cells. They display strong adsorption coefficients in the visible range of the sunlight in comparison with diamond silicon and remarkable themodynamical stability superior to most of the previously proposed direct or quasi-direct band gap silicon allotropes such as $\mathrm{Si}_{20}-\mathrm{T}^{5}$, Q135 mC12-Si ${ }^{6}$. Especially, the direct band gap M585-silicon possessing energy only $25 \mathrm{meV}$ per atom higher than that of diamond silicon and about $80 \mathrm{meV}$ lower than that of the recently synthesized $\mathrm{Si}_{24}{ }^{8}$ is expected to be experimentally produced for thin-film solar cells.

This work is supported by the National Natural Science Foundation of China (Grant Nos. A040204 and 11204261), the National Basic Research Program of China (2012CB921303 and 2015CB921103), the Hunan Provincial Innovation Foundation for Postgraduate (Grant No. CX2013A010), the Young Scientists Fund of the National Natural Science Foundation of China (Grant No. 11204260), and the Program for Changjiang Scholars and Innovative Research Team in University (IRT13093).
* Electronic address: jxzhong@xtu.edu.cn

1 W. Bludau, A. Onton, and W. Heinke, J. Appl. Phys. 45, 1846 (1974).

2 P. Lautenschlager,M. Garriga, L. Vina, andM. Cardona, Phys. Rev. B 36, 4821 (1987).

3 S. Botti, J. A. Flores-Livas, M. Amsler, S. Goedecker, and M. A. L. Marques, Phys. Rev. B 86, 121204 (2012).

4 J. Lv, Y. Wang, L. Zhu, and Y. Ma, Phys. Rev. Lett. 106, 015503 (2011); H. Wang, J. S. Tse, K. Tanaka, T. Iitaka, and Y. Ma, Proc. Natl. Acad. Sci. U.S.A. 109, 6463 (2012); Y. Wang, H. Liu, J. Lv, L. Zhu, H. Wang, and Y. Ma, Nat. Commun. 2, 563 (2011).

${ }^{5}$ H. J. Xiang, B. Huang, E. Kan, S.-H. Wei, and X. G. Gong, Phys. Rev. Lett. 110, 118702 (2013).

${ }^{6}$ Q. Q. Wang, B. Xu, J. Sun, H. Y. Liu, Z. S. Zhao, D. L. Yu, C. Z. Fan, and J. L. He, J. Am. Chem. Soc. 136, 9826 (2014).

7 I. H. Lee, J. Y. Lee, Y. J. Oh, S. Y. Kim, and K. J. Chang, Phys. Rev. B 90, 115209 (2011).

${ }^{8}$ D. Y. Kim, S. Stefanoski, O. O. Kurakevych, and T. A. Strobel, Nat. mater. doi:10.1038/nmat4140 (2014).

9 J. C. Conesa, J. Phys. Chem. B 106, 3402 (2002).

${ }^{10}$ G. Ceder and K. Persson, Sci. Am. 309, 36 (2013).

11 C. Y. He, L. Z. Sun, C. X. Zhang, X. Y. Peng, K. W. Zhang and J. X. Zhong, Phys. Chem. Chem. Phys. 14, 8410 (2012).
12 C. Y. He, L. Z. Sun, C.X. Zhang, X. Y. Peng, K. W. Zhang, and J. X. Zhong, Solid state commun. 152, 1560 (2012).

13 I. A. Baburin, D. M. Proserpio, V. A. Saleev, and A. V. Shipilova, Phys. Chem. Chem. Phys. 17, 1332 (2015).

${ }^{14}$ F. Wu, D. Jun, E. J. Kan, and Z. Y. Li, Solid. state. commun. 151, 1228 (2011).

15 K. Umemoto, R. M. Wentzcovitch, S. Saito, T. Miyake, Phys. Rev. Lett. 104, 125504 (2010).

${ }^{16}$ Q. Li, Y. M. Ma, A. R. Oganov, H. B. Wang, H. Wang, Y. Xu, T. Cui, H. K. Mao and G. T. Zou, Phys. Rev. Lett. 102, 175506 (2009).

17 J. H. Zhai, D. L. Yu, K. Luo, Q. Q. Wang, Z. S. Zhao, J. L. He, and Y. J. Tian, J.Phys.: Condens. Matter 24, 405803 (2012).

18 M. Amsler, J. A. Flores-Livas, L. Lehtovaara, F. Balima, S. A. Ghasemi, D. Machon, S. Pailhès, A. Willand, D. Caliste, S. Botti, A. S. Miguel, S. Goedecker and M. A. L. Marques, Phys. Rev. Lett. 108, 065501 (2012).

19 Z. S. Zhao, B. Xu, X. F. Zhou, L. M. Wang, B. Wen, J. L. He, Z. Y. Liu, H. T. Wang and Y. J. Tian, Phys. Rev. Lett. 107, 215502 (2011).

20 D. M. Ceperley and B. J. Alder, Phys. Rev. Lett. 45, 566 (1980).

21 J. P. Perdew and A. Zunger, Phys. Rev. B 23, 5048 (1981)

${ }^{22}$ G. Kresse and J. Furthmüller, Phys. Rev. B 54, 11169 (1996). 
23 G. Kresse and J. Furthmüller, Comput. Mater. Sci. 6, 15 (1996).

24 P. E. Blöchl, Phys. Rev. B 50, 17953 (1994).

25 G. Kresse and D. Joubert, Phys. Rev. B 59, 1758 (1999).

${ }^{26}$ K. Parlinski, Z-. Q. Li, and Y. Kawazoe, Phys. Rev. Lett. 78, 4063 (1997).

27 J. Heyd, G. E. Scuseria, and M. Ernzerhof, J. Chem. Phys. 118, 8207 (2003).

28 ASTM Standard G173-03(2008), Standard Tables for Reference Solar Spectral Irradiances: Direct Normal and Hemispherical on 37. Tilted Surface (ASTM International, West Conshohocken, PA, 2008).

${ }^{29}$ R. G. Little and M. J. Nowlan, Prog. Photovoltaics 5, 309 (1997); K. L. Chopra, P. D. Paulson, and V. Dutta, Prog. Photovoltaics 12, 69 (2004).

${ }^{30}$ F. P. Bundy, J. Chem. Phys. 46, 3437 (1967); A. F. Goncharov, I. N. Makarenko, and S. M. Stishov, Sov. Phys. JETP 69, 380 (1989); M. Hanfland, H. Beister, and K. Syassen, Phys. Rev. B 39, 12598 (1989);W. Utsumi and T. Yagi, Science 252, 1542 (1991); W. L. Mao, H. Mao, P. J. Eng, T. P. Trainor, M. Newville, C. C. Kao, D. L. Heinz, J. F. Shu, Y. Eng and R. J. Hemley, Science 302,
425 (2003).

31 Y. K. Vohra, K. E. Brister, S. Desgreniers, A. L. Ruoff, K. J. Chang, and M. L. Cohen, Phys. Rev. Lett. 561944 (1986); X. J. Chen, C. Zhang, Y. Meng, R. Q. Zhang, H. Q. Lin, V. V. Struzhkin, H. K. Mao, Phys. Rev. Lett. 106, 135502 (2011); J. Behler, A. K. R. Marton, D. Donadio, and M. Parrinello, Phys. Rev. Lett. 100, 185501 (2008); J. Z. Hu, L. D. Merkle, C. S. Menoni, and I. L. Spain, Phys. Rev. B 34, 4679 (1986).

32 Y. X. Zhao, F. Buehler, J. R. Sites, and I. L. Spain, Solid State Commun. 59, 679 (1986).

33 R. J. Needs and A. Mujica, Phys. Rev. B 51, 9652 (1995); B. G. Pfrommer, M. Côté, S. G. Louie, and M. L. Cohen, Phys. Rev. B 56, 6662 (1997); A. Mujica, A. Rubio, A.Muñoz, and R. J. Needs, Rev. Mod. Phys. 75, 863 (2003); Y. B. Gerbig, C. A. Michaels, A. M. Forster, and R. F. Cook, Phys. Rev. B 85, 104102 (2012).

${ }^{34}$ S. Ganguly, N. Kazem, D. Carter, and S. M. Kauzlarich, J. Am. Chem. Soc. 136, 1269 (2014).

${ }^{35}$ F. Kiefer, A. J. Karttunen, M. Döblinger, and T. F. Fässler, Chem. Mater. 23, 4578 (2011). 